\title{
Pkh1p-Ypk1p and Pkh1p-Sch9p Pathways Are Activated by Acetic Acid to Induce a Mitochondrial-Dependent Regulated Cell Death
}

\author{
António Rego $\mathbb{D}^{1,},{ }^{1,2}$ Filipa Mendes $\mathbb{D},{ }^{1}$ Vítor Costa $\mathbb{D}^{2,3,4}$ Susana Rodrigues Chaves $\mathbb{D}^{1}{ }^{1}$ \\ and Manuela Côrte-Real iD ${ }^{1}$ \\ ${ }^{1}$ Departamento de Biologia, Centro de Biologia Molecular e Ambiental, Universidade do Minho, Braga, Portugal \\ ${ }^{2}$ Instituto de Investigação e Inovação em Saúde, Universidade do Porto, Porto, Portugal \\ ${ }^{3}$ Instituto de Biologia Molecular e Celular, Universidade do Porto, Porto, Portugal \\ ${ }^{4}$ Departamento de Biologia Molecular, Instituto de Ciências Biomédicas Abel Salazar, Universidade do Porto, Porto, Portugal
}

Correspondence should be addressed to Manuela Côrte-Real; mcortereal@bio.uminho.pt

Received 17 October 2019; Revised 20 February 2020; Accepted 28 February 2020; Published 2 April 2020

Academic Editor: Ralf Braun

Copyright (C) 2020 António Rego et al. This is an open access article distributed under the Creative Commons Attribution License, which permits unrestricted use, distribution, and reproduction in any medium, provided the original work is properly cited.

\begin{abstract}
The yeast Saccharomyces cerevisiae undergoes a mitochondrial-dependent regulated cell death (RCD) exhibiting typical markers of mammalian apoptosis. We have previously shown that ceramide production contributes to RCD induced by acetic acid and is involved in mitochondrial outer membrane permeabilization and cytochrome $c$ release, especially through hydrolysis of complex sphingolipids catalyzed by Isc1p. Recently, we also showed that Sch9p regulates the translocation of Isc1p from the endoplasmic reticulum into mitochondria, perturbing sphingolipid balance and determining cell fate. In this study, we addressed the role of other signaling proteins in acetic acid-induced RCD. We found that single deletion of PKH1 or YPK1, as shown for SCH9 and ISC1, leads to an increase in cell survival in response to acetic acid and that Pkh1/2p-dependent phosphorylation of Ypk1p and Sch9p increases under these conditions. These results indicate that Pkh1p regulates acetic acid-induced RCD through Ypk1p and Sch9p. In addition, our results suggest that Pkh1p-Ypklp is necessary for isc1 $\Delta$ resistance to acetic acid-induced RCD. Moreover, double deletion of ISC1 and PKH1 has a drastic effect on cell survival associated with increased ROS accumulation and release of cytochrome $c$, which is counteracted by overexpression of the PKA pathway negative regulator PDE2. Overall, our results suggest that Pkh1p-Ypk1p and Pkh1p-Sch9p pathways contribute to RCD induced by acetic acid.
\end{abstract}

\section{Introduction}

Sphingolipids are essential components of all eukaryotic membranes. In addition to their structural function, sphingolipids play important roles in cellular responses to many different stimuli, acting as messengers in a variety of signaling pathways. From yeast to higher eukaryotes, sphingolipids are involved in the regulation of cell growth, apoptosis, cell cycle arrest, cell wall integrity, nutrient uptake, and longevity $[1,2]$. Moreover, sphingolipids have been associated with several human pathologies linked to apoptosis dysfunction, such as cancer and neurodegenerative diseases [3]. Consequently, understanding how signaling pathways regulate sphingolipid metabolism and cell death will provide new hints to the elucidation of molecular mechanisms underlying these diseases.
Sphingolipid metabolism is highly complex and interconnected and its regulation is of fundamental importance for cells due to their relevant role in cellular homeostasis [4]. Therefore, cells possess several mechanisms that contribute to the control of enzymes at different steps of sphingolipid synthesis and breakdown to adjust the levels of individual sphingolipids. However, in response to stress conditions, many biosynthetic intermediates can unduly accumulate and severely influence cell functions and fate. Based on several observations, it is now established that sphingolipids play a role in apoptosis [5]. Exposure of yeast cells to acetic acid has been shown to trigger a regulated cell death (RCD) process with features similar to mammalian apoptosis, such as exposure of phosphatidylserine to the outer leaflet of the plasma membrane, alterations in mitochondrial structure, chromatin condensation, nuclear DNA fragmentation, 


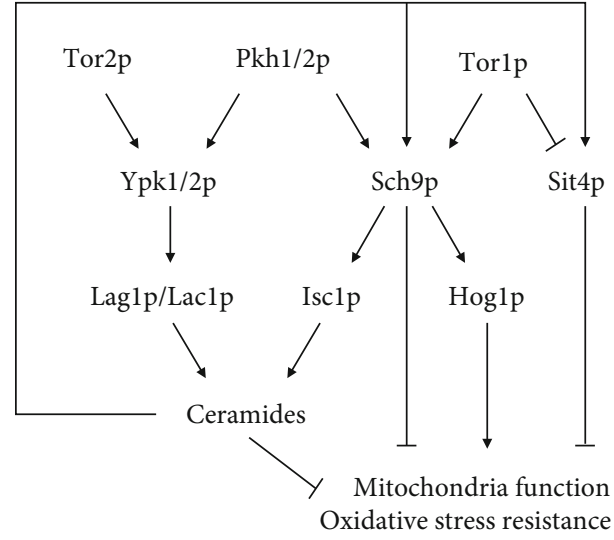

FIGURE 1: Schematic overview of the signaling pathways involved in sphingolipid metabolism, mitochondrial function, and oxidative stress resistance. Ypklp is the sole paralog responsible for the phosphorylation of ceramide synthases Laclp and Laglp, establishing the link between this kinase and sphingolipids [26]. Sch9p functions as the major gatekeeper of sphingolipid homeostasis, as a feedback system complementary to ceramide production in response to both internal sphingolipid signals and nutrient availability and for the proper translocation of Isc1p from the ER to mitochondria during the diauxic shift [27]. Recently, we showed that Sch9p modulates acetic acid-induced mitochondrial cell death through the regulation of Isclp cellular distribution, thus affecting the sphingolipid balance and ultimately regulating cell fate [28].

caspase-like activation, reactive oxygen species (ROS) accumulation, cytochrome $c$ release, and mitochondrial dysfunction [6-10]. An increase in ceramide levels during apoptosis has been also reported in response to a variety of stimuli and in different cell types. In particular, we have reported the involvement of ceramide metabolism in cell death induced by acetic acid. We showed that isc1 $\Delta$ and lag $1 \Delta$ mutants, lacking inositol phosphosphingolipid phospholipase $\mathrm{C}$ and ceramide synthase, respectively, exhibited a higher resistance to acetic acid that was associated with a decrease in some dihydroceramide and phytoceramide species. Accordingly, these mutants also displayed lower levels of ROS accumulation and reduced mitochondrial alterations, which led us to conclude that acetic acid increases ceramide levels through hydrolysis of complex lipids and de novo synthesis catalyzed by Isclp and Lag1p, respectively, leading to a mitochondriamediated RCD [11]. However, several important questions related to this model system remained to be elucidated, including which signaling pathways are involved in the activation of these enzymes in the course of acetic acid-induced cell death.

An important regulator of the yeast sphingolipid biosynthetic pathway, which controls the sphingolipid balance, is the serine/threonine kinase Pkh1p (Figure 1). Pkh1p, and its paralog $\mathrm{Pkh} 2 \mathrm{p}$, is an orthologue of the mammalian 3-phosphoinositide-dependent kinase PDK1 [12]. Four protein kinase substrates of Pkh1p and/or Pkh2p have already been identified as Pkc1p [13, 14], Ypk1p, Ypk2p, and Sch9p $[12,15]$. In particular, the serine/threonine protein kinases Ypk1/2p, orthologues of the mammalian protein kinase serum- and glucocorticoid-inducible protein kinase (SGK), are phosphorylated and activated by Pkh1/2p; Pkh1p preferentially activates Ypk1p and Pkh2p preferentially activates Ypk2p [16]. Ypk1p activation also requires its phosphorylation by the Target of Rapamycin (TOR) complex 2 (TORC2) [17]. Sch9p can be phosphorylated by Pkh1/2p at the PDK1 site in its activation loop $[18,19]$ or by TORC1 (TOR complex 1) at multiple C-terminal sites [20]. A recent work showed that Sch9p kinase is also necessary for yeast to properly adapt to hyperosmotic stress, at least in part, through the Hog1p MAPK complex [21]. In yeast, Sit4p is the catalytic subunit of the ceramide-activated protein phosphatase (CAPP) [22]. This phosphatase plays a key regulator role in sphingolipid metabolism by acting downstream of TORC1 signaling. It was shown that TORC1 can downregulate Sit $4 p$ activity through the phosphorylation of Tap42p, which then inhibits Sit4p [23], a mechanism necessary to activate complex sphingolipid biosynthesis [24, 25].

In this study, we investigated the interplay between these signaling networks in the regulation of acetic acid-induced RCD. We show that Pkh1p-Ypklp and Pkh1p-Sch9p pathways are activated during acetic acid exposure to induce cell death. In addition, we explored how these signaling pathways affect the resistance phenotype of the isc $1 \Delta$ mutant to acetic acid.

\section{Material and Methods}

2.1. Yeast Strains, Plasmids, and Growth Conditions. All Saccharomyces cerevisiae strains used in this study are listed in Table 1. The yeast S. cerevisiae strain BY4741 was used throughout this study as the wild-type strain. The isc1 $\Delta$ $y p k 1 \Delta$ mutant was constructed by homologous recombination of a disruption cassette containing the flanking regions of YPK1 (YPK1::KanMX4), which was amplified by Polymerase Chain Reaction (PCR) using genomic DNA isolated from the $y p k 1 \Delta$ mutant, into the BY4741 isc1 $1:: L E U 2$ strain. After transformation using the LiAc method [29], mutants were selected on rich medium (YPD; $1 \%(w / v)$ yeast extract, $2 \%$ $(w / v)$ bactopeptone, and $2 \%(w / v)$ glucose) containing $200 \mu \mathrm{g} / \mathrm{mL}$ geneticin, and proper integration of the cassette was confirmed by PCR. The isc $1 \Delta$ pkh1 $\Delta$ double mutant was generated by sporulation of the diploids resultant from

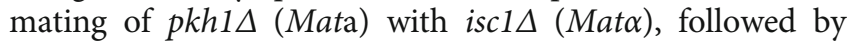
tetrad dissection by standard techniques.

Strains were grown in Synthetic Complete Galactose medium (SC Gal; 2\% $(w / v)$ Galactose, $0.67 \%(w / v)$ yeast nitrogen base without amino acids, $0.14 \%(w / v)$ drop-out mixture lacking histidine, leucine, tryptophan, and uracil, $0.008 \%(w / v)$ histidine, $0.04 \%(w / v)$ leucine, $0.008 \%(w / v)$ tryptophan, and $0.008 \%(w / v)$ uracil) at $26^{\circ} \mathrm{C}$ in an orbital shaker at $200 \mathrm{rpm}$, with a ratio of flask volume/medium of $5: 1$. Strains transformed with the indicated plasmids were grown in the same medium lacking the appropriate amino acids. Galactose was used as the carbon and energy source as it is less effective in the repression of respiratory metabolism, leading to higher mitochondrial mass and facilitating the assessment of the involvement mitochondria in different cell processes [30]. We routinely confirm 
TABLE 1: List of S. cerevisiae strains used in this study.

\begin{tabular}{|c|c|c|}
\hline Strain & Genotype & Source \\
\hline BY4741 & MATa, his3-1, leu2-0, met15-0, ura3-0 & Euroscarf \\
\hline BY4741 pYES2 & BY4741 harboring pYES2 & This study \\
\hline BY4741 pISC1-FLAG & BY4741 harboring pYES2-ISC1-FLAG & This study \\
\hline BY4741 pRS315 & BY4741 harboring pRS315 & This study \\
\hline BY4741 pRS316 & BY4741 harboring pRS316 & This study \\
\hline BY4741 pRS426 & BY4741 harboring pRS426 & This study \\
\hline BY4741 pPDE2 & BY4741 harboring pRS426-PDE2 & This study \\
\hline isc $1 \Delta$ & BY4741 isc1 $\triangle:: L E U 2$ & {$[31]$} \\
\hline isc1 $1 \Delta \mathrm{pRS} 426$ & isc1 $\Delta$ harboring pRS426 & This study \\
\hline$i s c 1 \triangle \mathrm{p} P D E 2$ & isc $1 \Delta$ harboring pRS426-PDE2 & This study \\
\hline tor1 $1 \Delta$ & BY4741 tor $1 \Delta:: K a n M X 4$ & Euroscarf \\
\hline pkh1s & BY4741 pkh1 $1:$ KanMX4 & Euroscarf \\
\hline pkh1 1 pRS426 & pkh1 $\Delta$ harboring pRS426 & This study \\
\hline$p k h 1 \triangle \mathrm{p} P D E 2$ & pkh1 $\Delta$ harboring pRS426-PDE2 & This study \\
\hline$i s c 1 \Delta p k h 1 \Delta$ & BY4741 isc1 $1:: L E U 2$ pkh1 $1::$ KanMX4 & This study \\
\hline$i s c 1 \Delta p k h 1 \Delta$ pRS426 & isc1 $1 \Delta$ ph $1 \Delta$ harboring pRS426 & This study \\
\hline$i s c 1 \triangle p k h 1 \triangle \mathrm{p} P D E 2$ & $i s c 1 \Delta p k h 1 \Delta$ harboring pRS426-PDE2 & This study \\
\hline$y p k 1 \Delta$ & BY4741 ypk1 $\Delta::$ KanMX4 & Euroscarf \\
\hline$y p k 1 \Delta \mathrm{pRS} 316$ & $y p k 1 \Delta$ harboring pRS316 & This study \\
\hline$y p k 1 \Delta \mathrm{p} Y P K 1$ & $y p k 1 \Delta$ harboring pRS316-YPK1 & This study \\
\hline$y p k 1 \triangle \mathrm{p} Y P K 1(\mathrm{~T} 504 \mathrm{D})$ & $y p k 1 \Delta$ harboring pRS316-YPK1(T504D) & This study \\
\hline$i s c 1 \Delta y p k 1 \Delta$ & BY4741 isc1 $1::$ LEU2 ypk1 $1::$ KanMX4 & This study \\
\hline $\operatorname{sch} 9 \Delta$ & BY4741 sch9 $9:: K a n M X 4$ & Euroscarf \\
\hline $\operatorname{sch} 9 \Delta \mathrm{pRS} 315$ & sch $9 \Delta$ harboring pRS315 & This study \\
\hline sch $9 \Delta \mathrm{pSCH} 9$ & sch $9 \Delta$ harboring pRS315-SCH9 & This study \\
\hline sch9D pSCH9(T570A) & sch9 4 harboring pRS315-SCH9(T570A) & This study \\
\hline sit4 $\Delta$ & BY4741 sit4A::KanMX4 & Euroscarf \\
\hline $\operatorname{hog} 1 \Delta$ & BY4741 hog1 $\Delta:: K a n M X 4$ & Euroscarf \\
\hline
\end{tabular}

the same phenotypes in glucose- and galactose-grown cells and have shown in a previous study that translocation of Isclp to mitochondria in response to acetic acid occurs similarly in glucose- and galactose-grown cells [28].

2.2. Cell Death Assays. For acetic acid treatments, exponential phase cells $\left(\mathrm{OD}_{600}=0.2-0.5\right)$ grown as described before were harvested and suspended in SC Gal at pH3.0 (set with $\mathrm{HCl}$ ) without or with $140 \mathrm{mM}$ acetic acid (Panreac, Spain). Cell viability was measured as a percentage of colonyforming units (c.f.u.) on YPD plates.

2.3. Assessment of Plasma Membrane Integrity. Plasma membrane integrity was assessed by flow cytometry using propidium iodide (PI, Sigma-Aldrich) staining. PI was added to yeast cell suspensions to a final concentration of $5 \mu \mathrm{g} / \mathrm{mL}$ and incubated for $10 \mathrm{~min}$ at room temperature. Cells with red fluorescence (FL-3 channel $(488 / 620 \mathrm{~nm})$ ) were considered to have lost their plasma membrane integrity.

2.4. Assessment of ROS Accumulation. Intracellular superoxide anion was detected by flow cytometry using dihydroethidium (DHE, Molecular Probes, Eugene, USA). Untreated or acetic acid-treated cells were harvested by centrifugation, suspended in PBS $\left(80 \mathrm{mM} \mathrm{Na} \mathrm{HPO}_{4}, 20 \mathrm{mM}\right.$ $\mathrm{NaH}_{2} \mathrm{PO}_{4}$, and $100 \mathrm{mM} \mathrm{NaCl}$ ), and incubated with $5 \mu \mathrm{g} / \mathrm{mL}$ $\mathrm{DHE}$ for $30 \mathrm{~min}$ in the dark. Cells with red fluorescence (FL-3 channel (488/620 nm)) were considered to accumulate superoxide anion.

2.5. Determination of Oxygen Consumption Rates. Yeast strains were cultured under the conditions described above, harvested, and suspended in water $\left(\mathrm{OD}_{600 \mathrm{~nm}}=20\right)$. A Clark electrode connected to a Yellow Spring Instruments 5300 biological oxygen monitor and to a Kipp \& Zonen flatbed chart recorder was used. The electrode was immersed in a chamber with $4.6 \mathrm{~mL}$ of deionized water stirred with a magnetic bar. The Clark electrode was calibrated at time 0 for $100 \%$ oxygen saturation. A volume of $0.4 \mathrm{~mL}$ of yeast suspension was added to the chamber, and the oxygen consumption was recorded. The oxygen consumption rate values were calculated from the slopes of the initial part of the oxygen consumption trace and normalized to the dry weight of the respective cell culture. 
2.6. Determination of Protein Phosphorylation Levels. For phosphorylation detection, cells were grown and treated under the conditions described above, harvested at the indicated times, and processed. Briefly, cells were immediately centrifuged and incubated in $0.25 \mathrm{M} \mathrm{NaOH}$ for $5 \mathrm{~min}$ at room temperature. After centrifugation, the pellet was suspended in SDS sample buffer $(3 \%(w / v)$ DTT, $125 \mathrm{mM}$ Tris, 20\% ( $v / v)$ Glycerol, 0.001\% ( $w / v)$ Bromophenol Blue, and $4 \%(w / v)$ SDS $)$ and boiled at $95^{\circ} \mathrm{C}$ for $5 \mathrm{~min}$. Samples were then separated electrophoretically on a $10 \%$ SDS-polyacrylamide gel and transferred to a nitrocellulose membrane (GE Healthcare) at $0.8 \mathrm{~mA} / \mathrm{cm}^{2}$ for $1 \mathrm{~h}$ and $30 \mathrm{~min}$. The membrane was blocked in TBS-T $(20 \mathrm{mM}$ Tris, $140 \mathrm{mM} \mathrm{NaCl}$, and $0.05 \%(v / v)$ Tween-20 pH7.6) containing 5\% $(w / v)$ BSA. Membranes were cut into strips and incubated with the primary antibodies mouse monoclonal anti-yeast phosphoglycerate kinase (PGK1) antibody (1:5000, Molecular Probes), rabbit anti-Sch9p antibody $(1: 1000)$, goat anti-Ypklp antibody $(1: 100$, Santa Cruz Biotechnology), rabbit anti-Phospho-T570-Sch9p antibody (1:10000), rabbit anti-Phospho-T504 Ypk1p (1:1000, Cell Signaling Technology, Phospho-PKC (pan) (zeta Thr410) (190D10)), and rabbit anti-Phospho-Slt2p (1:1000, Cell Signaling, anti-Phospho-p44/42 MAPK (Erk1/2) (Thr202/Tyr204)), followed by incubation with secondary antibodies against mouse, rabbit, or goat IgG peroxidase (1:5000; Sigma-Aldrich). Immunodetection of bands was revealed by chemiluminescence (ECL, GE Healthcare).

\subsection{Immunoprecipitation Analysis. BY4741 cells expressing} Isclp-FLAG or vector control were grown in SC Gal medium without uracil, harvested at early exponential phase, and washed and suspended in IP buffer $(0.5 \%(v / v)$ Triton X$100,150 \mathrm{mM} \mathrm{NaCl}, 1 \mathrm{mM}$ EDTA, $50 \mathrm{mM}$ Tris $\mathrm{pH} 7.4,1 \mathrm{x}$ Complete Mini Protease Inhibitor Cocktail (Roche)). Cells were lysed for $10 \mathrm{~min}$ (short pulses of $1 \mathrm{~min}$ with $1 \mathrm{~min}$ intervals on ice) by vigorous shaking of the cell suspension in the presence of glass beads, and total protein concentration was assessed by the Bradford method using bovine serum albumin as a standard. $1000 \mu \mathrm{g}$ of soluble protein were immunoprecipitated overnight at $4^{\circ} \mathrm{C}$ with $1 \mu \mathrm{L}$ of mouse $\alpha$ FLAG M2 antibody (Sigma-Aldrich). Then, $20 \mu \mathrm{L}$ of Protein G Sepharose (Sigma-Aldrich) were added and the suspension incubated for 2 hours at $4^{\circ} \mathrm{C}$, with agitation. The beads were sedimented, and the unbound proteins were removed by three washing steps with IP buffer and collected by centrifugation. Bead-bound immune complexes were solubilized with SDS sample buffer and boiled for $5 \mathrm{~min}$, and the supernatant was analyzed by Western blot as previously described using the primary antibodies goat anti-Ypk1p antibody (1:100, Santa Cruz Biotechnology) and mouse anti-FLAG M2 (1:5000, Sigma-Aldrich), followed by incubation with secondary antibodies anti-goat IgG-peroxidase (1:5000; Sigma-Aldrich) and anti-mouse IgG-peroxidase TrueBlot ${ }^{\circledR}$ ULTRA (1:5000; Rockland), respectively.

2.8. Cytochrome $c$ Release. For cytochrome $c$ detection, cells were grown and treated under the conditions described above, harvested at early exponential phase, and suspended in suspension buffer (1.2 M sorbitol, $0.06 \mathrm{M}$ Sodium phosphate (pH 7.5), and 0.01 M EDTA). Cells were then digested with Zymolyase $20 \mathrm{~T}$ (ImmunO, MP Biomedicals) to obtain spheroplasts, washed twice with $1.2 \mathrm{M}$ sorbitol, and suspended in lysis buffer $(0.5 \mathrm{M}$ Sorbitol, $20 \mathrm{mM}$ Tris/HCl (pH 7.5), and $1 \mathrm{mM} \mathrm{EDTA).} \mathrm{Spheroplasts} \mathrm{were} \mathrm{lysed} \mathrm{with} \mathrm{a}$ few strokes in a glass Dounce homogenizer (tight fitting piston). Homogenates were then centrifuged at $2500 \mathrm{rpm}$ for $10 \mathrm{~min}$, and the supernatant was centrifuged at $15000 \mathrm{rpm}$ for $15 \mathrm{~min}$. The supernatant constitutes the cytosolic fraction. The pellet, containing the mitochondrial fraction, was suspended in lysis buffer and both fractions frozen in liquid nitrogen and stored at $-80^{\circ} \mathrm{C}$.

Mitochondrial and cytosolic fractions were separated electrophoretically on a $12.5 \%$ SDS-polyacrylamide gel and transferred to a Hybond-P polyvinylidene difluoride membrane (PVDF; GE Healthcare) at $0.8 \mathrm{~mA} / \mathrm{cm}^{2}$ for $1 \mathrm{~h}$. Membranes were blocked for $1 \mathrm{~h}$ in PBS-T (PBS with $0.05 \%(v / v)$ Tween-20) containing 5\% $(w / v)$ nonfat dry milk, cut into strips, and incubated with the primary antibodies mouse monoclonal anti-yeast phosphoglycerate kinase (PGK1) antibody (1:5000, Molecular Probes), mouse monoclonal antiyeast porin (POR1) antibody (1:10000, Molecular Probes), or rabbit polyclonal anti-yeast cytochrome $c$ (CYC1) antibody ( $1: 2000$, custom-made by MilleGen), followed by incubation with secondary antibodies against mouse or rabbit IgG-peroxidase (1:5000; Sigma-Aldrich). Immunodetection of bands was revealed by chemiluminescence (ECL, GE Healthcare).

2.9. Calcofluor White Staining. Yeast cells were grown under the conditions described above, harvested at early exponential phase, suspended in PBS, incubated with $0.02 \%(w / v)$ Calcofluor White (CFW, Fluka, St. Louis, MO) for $30 \mathrm{~min}$ at room temperature, and washed 3 times with PBS. Cells were observed using a Leica Microsystems DM-5000B epifluorescence microscope with appropriate filter settings using a 100x oil-immersion objective. Images were acquired with a Leica DCF350FX digital camera and processed with LAS AF Leica Microsystems software.

\section{Results and Discussion}

3.1. Pkh1p, Ypk1p, and Sit4p Regulate Acetic Acid-Induced Cell Death. The involvement of sphingolipid signaling in the regulation of biological processes, such as cell death, has been addressed in both mammals and yeast [4]. In yeast, several signaling pathways have been connected with Isclp function in the response to several stimuli, like hydrogen peroxide and ageing [31-35]. We therefore aimed to determine the involvement of those signaling pathways in acetic acidinduced RCD. We first assessed the relative contribution of Torlp, Pkh1p, Ypk1p, Hoglp, and Sit4p to acetic acidinduced RCD under our experimental conditions, and compared with that of Sch9p and Isc1p [28]. For this purpose, S. cerevisiae BY4741 and isc $1 \Delta$, tor $1 \Delta$, pkh1 $1 \Delta, y p k 1 \Delta$, sch $9 \Delta$, sit $4 \Delta$, and hog $1 \Delta$ mutants were grown to exponential phase in SC Gal medium and exposed to $140 \mathrm{mM}$ acetic acid, pH 3.0, for 180 min (Figure 2). 


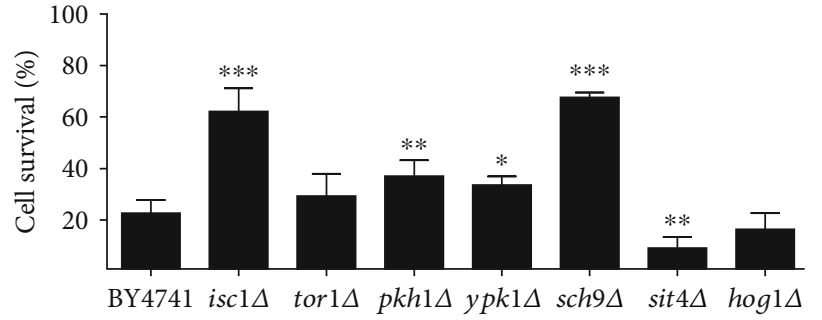

(a)

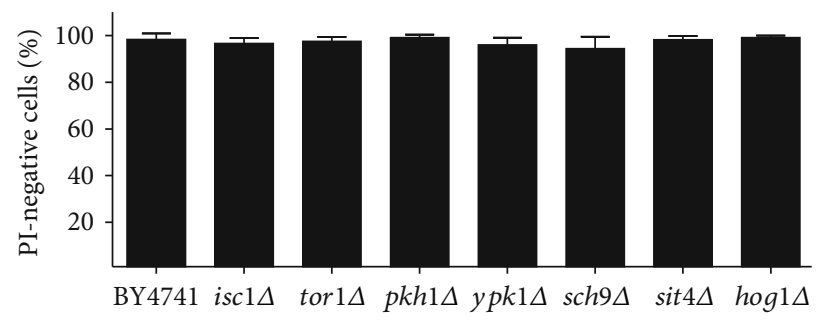

(b)

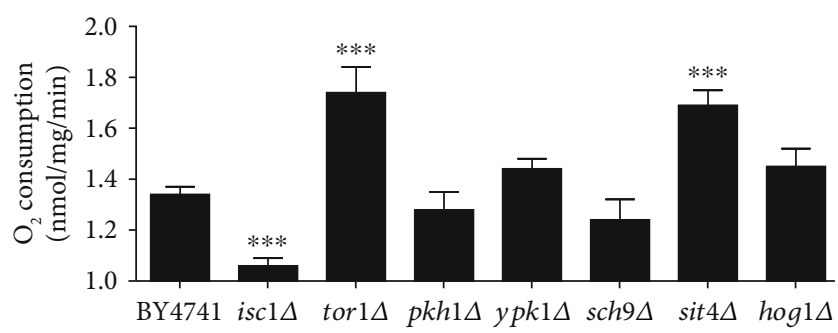

(c)

FIGURE 2: Pkh1p, Ypk1p, Sch9p, and Sit4p regulate acetic acid-induced cell death. (a) Cell survival of the indicated S. cerevisiae strains to $140 \mathrm{mM}$ of acetic acid, $\mathrm{pH} 3.0$, for $180 \mathrm{~min}$. Cell viability was determined by standard dilution plate counts and expressed as a percentage of c.f.u. on YPD plates in relation to time 0. (b) Percentage of PI-negative cells of the indicated S. cerevisiae strains after acetic acid exposure assessed by flow cytometry. (c) Basal $\mathrm{O}_{2}$ consumption rates of the indicated S. cerevisiae strains determined using a Clark electrode. Values represent mean $\pm S D$ of at least three independent experiments. Values significantly different from the BY4741 strain: ${ }^{*} P<0.05$, ${ }^{* *} P<0.01,{ }^{* * *} P<0.001$, one-way ANOVA, and Tukey's Test.

As shown in Figure 2(a), deletion of PKH1 or YPK1 increased the resistance of yeast cells to acetic acid, whereas deletion of SIT4 decreased the resistance and TOR1 or HOG1 deletion had no significant effect. Also, in accordance with our previous results, $\mathrm{ISC} 1$ and $\mathrm{SCH} 9$ deletion increased cell survival to a similar extent [28]. Acetic acid-induced cell death in all strains was not associated with significant loss of plasma membrane integrity as measured by propidium iodide (PI) staining, indicating this is a non-necrotic regulated event (Figure 2(b)). Since plasma membrane integrity was assessed after exposing cells to acetic acid for $180 \mathrm{~min}$, but viability assessed only $48 \mathrm{~h}$ after plating those cells on rich media, we sought to determine at what point during this period cells died, which ultimately results in plasma membrane disruption. For this purpose, after acetic acid treatment, wild-type cells were washed, suspended in fresh medium, and grown at $26^{\circ} \mathrm{C}$. Plasma membrane integrity was again measured by PI staining, and we found that about $90 \%$ of wild-type cells were PI positive after $240 \mathrm{~min}$ in fresh medium (Figure S1). Our results therefore show that cells that are exposed to acetic acid for $180 \mathrm{~min}$ reach the point of no return in the regulated cell death process, still maintaining plasma membrane integrity. Then, even after acetic acid is removed, they continue the cell death process and lose plasma membrane integrity and the ability to form colonies within $240 \mathrm{~min}$.

Cell death induced by acetic acid in S. cerevisiae is mediated by a mitochondria-dependent pathway and involves ROS accumulation, reduction of oxygen consumption, and translocation of cytochrome $c$ from the mitochondria to the cytosol [6]. In addition, respiratory-deficient mutants, namely $\rho^{0}$ cells lacking mitochondrial DNA, the null atp10 $\Delta$ mutant (deleted in an assembly factor of mitochondrial ATPase) and the null cyc3 3 mutant (deleted in the gene encoding a heme lyase) were described as more resistant to death induced by acetic acid [6]. We therefore sought to determine whether the phenotypes of the mutants described above correlated with changes in mitochondria respiration. For this purpose, cells were grown to exponential phase, and the basal oxygen consumption rate (OCR) was determined using a Clark electrode (Figure 2(c)). The effect of an uncoupler and respiratory chain inhibitors were used as controls to confirm that OCR mirrors mitochondria function (Figure S2). Deletion of TOR1 or SIT4 increased the OCR and deletion of ISC1 decreased OCR, whereas deletion of the other genes had no effect. Indeed, several acetic acidresistant mutants ( $p k h 1 \Delta, y p k 1 \Delta$, and $s c h 9 \Delta)$ exhibited an OCR similar to that of the wild-type. The increase of OCR in $\operatorname{tor} 1 \Delta$ cells was consistent with previous data showing that disruption of TORC1 signaling, via deletion of TOR1, relieves respiration repression by increasing mitochondrial translation, and consequently the number/activity of respiratory complexes [36]. Although Sit4p is negatively regulated by TORC1 signaling [37], previous studies also showed an increase of OCR in sit4 $\Delta$ mutants [31]. Overall, these results suggest that acetic acid resistance of the tested mutants is not associated with basal OCR levels.

In conclusion, besides Sch9p, we identified three new important players of several signaling pathways with a role in acetic acid-induced cell death, namely, Pkh1p and Ypklp with a pro-death and Sit4p with a pro-survival role.

3.2. Acetic Acid Induces Pkh1p-Dependent Phosphorylation of Sch $9 p$ and Ypklp. Given the phenotypic evidence that Pkhlp, 

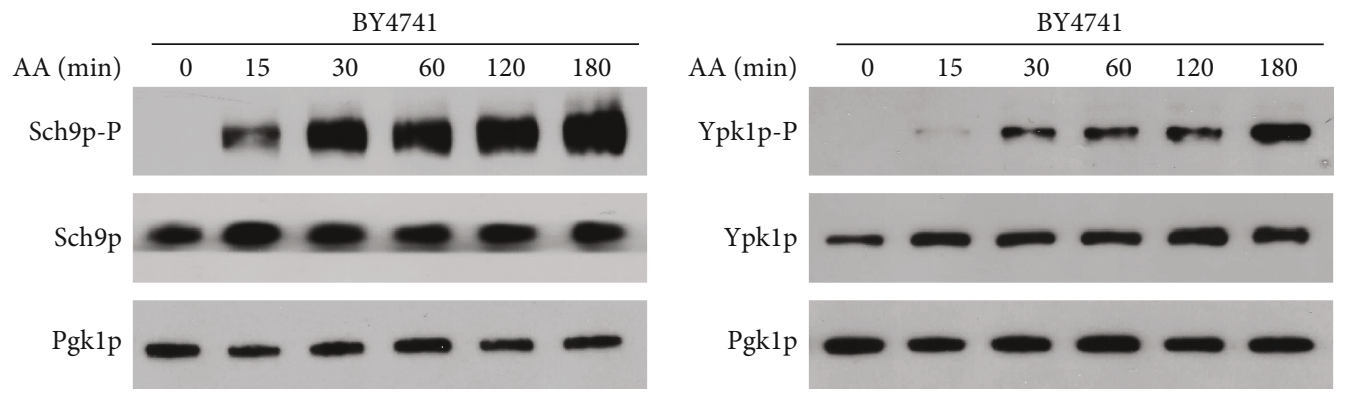

(a)
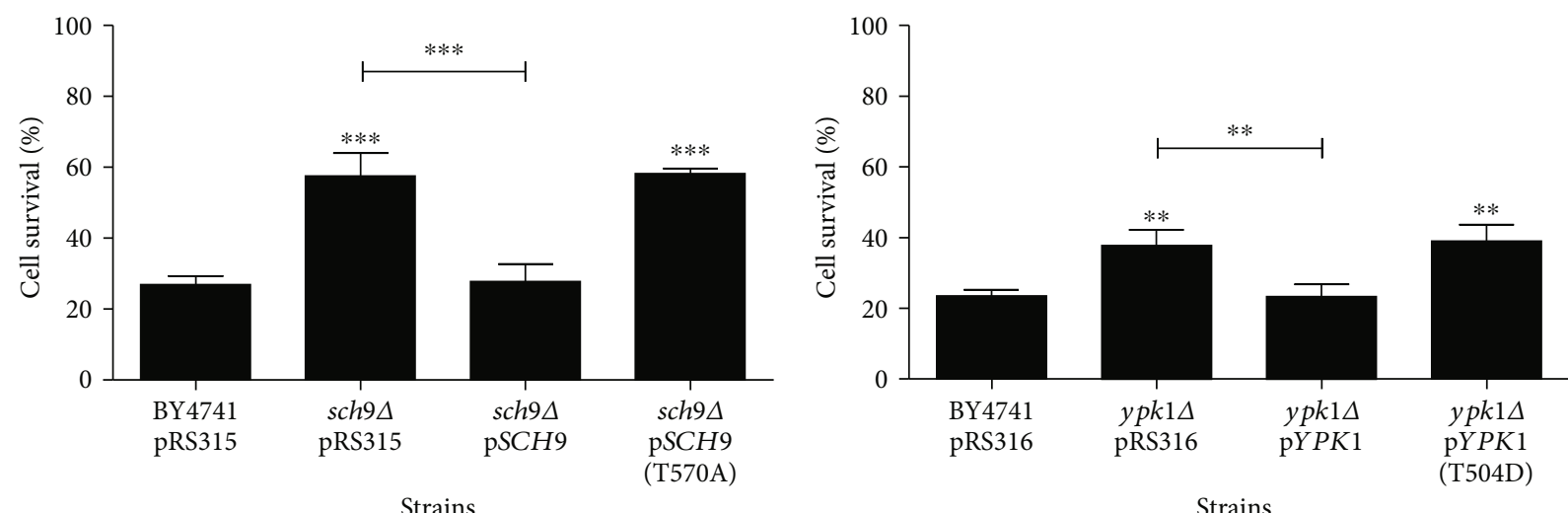

(b)

FIGURE 3: Acetic acid induces PDK1-dependent phosphorylation of Ypk1p and Sch9p. (a) Western blot analysis of the levels of total and phosphorylated Sch9p and Ypk1p in wild-type cells before and after exposure to acetic acid for up to 180 min. Cytosolic phosphoglycerate kinase (Pgk1p) levels were used as the loading control. Only a representative image of Pgk1p levels was represented in the figure, although the levels of Pgklp were evaluated in both blots of the total and phosphorylated proteins. (b) Cell survival of the indicated strains to $140 \mathrm{mM}$ of acetic acid, $\mathrm{pH} 3.0$, for $180 \mathrm{~min}$. Cell viability was determined by standard dilution plate counts and expressed as a percentage of c.f.u. on YPD plates in relation to time 0 . Values represent mean $\pm \mathrm{SD}$ of at least three independent experiments. Values significantly different from BY4741 or between the indicated strains: ${ }^{* *} P<0.01,{ }^{* * *} P<0.001$, one-way ANOVA, and Tukey's Test.

Ypk1p, and Sch9p have a pro-death role in acetic acidinduced apoptosis, we next analyzed whether the corresponding signaling pathways were activated. It has been demonstrated that the Pkhlp kinase can differentially activate the Sch9p kinase by phosphorylating its T570 residue $[18,19]$ or the Ypklp kinase by phosphorylating its T504 residue $[12,14]$. To determine whether Pkhlp activates Ypklp and/or Sch9p in response to acetic acid, phosphorylated and total protein levels of Sch9p and Ypk1p were analyzed (Figure 3(a)). For this purpose, we used phospho-sitedirected antibodies Phospho-PKC (pan) (zeta Thr410) (190D10) directed against the highly homologous PDK1 site in human SGK1, which specifically detects P-Thr504 in Ypk1p $[12,38]$ and anti-Phospho-T570-Sch9p, which specifically recognizes this PDK1 site in Sch9p [20].

Our data show that acetic acid exposure leads to phosphorylation of both Sch9p and Ypk1p at the PDK1 sites, without affecting the total levels of the corresponding proteins. An increase of phosphorylated Sch9p and Ypklp was already observed after $15 \mathrm{~min}$, but it further increased with the time of exposure to acetic acid. Phosphorylation of the single PDK1 site in Ypk1p or Sch9p is required for their function in vivo [18]. We further confirmed that expression of Sch9p (T570A) and Ypk1p (T504D) does not revert the resis- tant phenotype of the $s c h 9 \Delta$ and $y p k 1 \Delta$ mutants, respectively, while expression of wild-type Sch9p and Ypk1p renders cells more sensitive to acetic acid, as expected (Figure 3(b)). Though Sch9p can be phosphorylated at T570 by both Pkh1p and Pkh2p, Pkh2p has a minor contribution [20]. It has also been shown that Pkh1p preferentially activates Ypklp, both biochemically and genetically [16]. Therefore, while we cannot fully exclude a contribution of Pkh2p, our results indicate that Pkh1p phosphorylates Ypk1p and Sch9p to mediate acetic acid-induced RCD.

3.3. Pkh1p And Ypk1p Are Necessary for isc1 $1 \Delta$ Resistance to Acetic Acid-Induced RCD. Our data implicate the PkhlpSch9p and Pkh1p-Ypklp cascades in acetic acid-induced RCD in the wild-type strain. However, the results suggest that Pkh1p, Ypk1p, and Sch9p may modulate this process in a different manner, as acetic acid resistance of the correspondent mutants was for the most part very different (Figure 2(a)). This could be due to differential functional interplay between the different components of sphingolipid metabolism pathways, and in particular with Isclp. In line with this, our previous results demonstrated that acetic acid treatment induces Sch9p-dependent translocation of Isclp from endoplasmic reticulum to mitochondria [28]. However, 


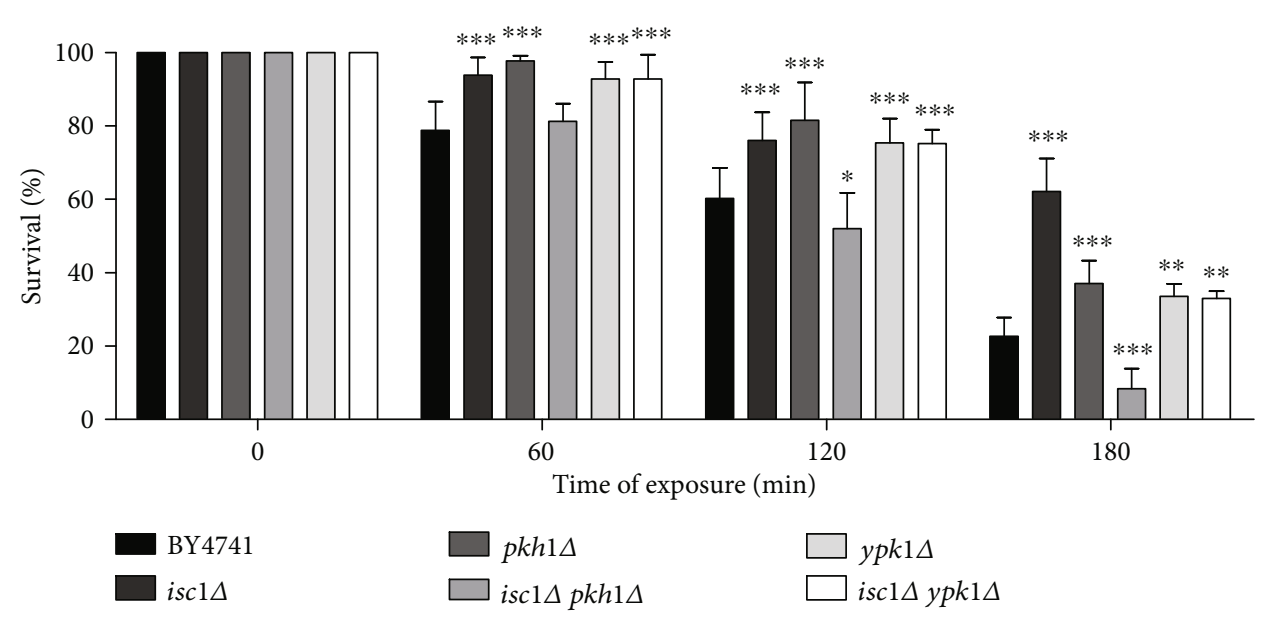

FIGURE 4: The Pkh1p-Ypk1p pathway is necessary for resistance of the isc1 $\Delta$ mutant to acetic acid-induced RCD. Cell survival of the indicated strains to $140 \mathrm{mM}$ of acetic acid, $\mathrm{pH} 3.0$, for $180 \mathrm{~min}$. Values represent mean \pm SD of at least three independent experiments. Values significantly different from BY4741: ${ }^{*} P<0.05,{ }^{* *} P<0.01,{ }^{* * *} P<0.001$, One-way ANOVA, and Tukey Test.

the higher survival of the $\operatorname{sch} 9 \Delta$ mutant compared to $p k h 1 \Delta$ suggests that Sch9p might also be activated by other kinases different from Pkh1p (possibly Pkh2p and others), and the phosphorylation of Ypk1p by Pkh1/2p suggests that these kinases have other functions independent of Sch9p in acetic acid-induced cell death.

Isclp function has been connected with several other signaling pathways involved in ceramide signaling. Indeed, it was demonstrated that deletion of SIT4 [31], HOG1 [33], or TOR1 [35] suppresses the phenotypes displayed by isc1 $\Delta$ cells. Since we found that the Pkhlp-Ypklp pathway is activated during acetic acid treatment and the survival phenotypes of the mutants were relatively different from isc $1 \Delta$, we assessed the effect of deleting PKH1 or YPK1 in the survival of the isc $1 \Delta$ mutant to acetic acid (Figure 4).

Deletion of either $P K H 1$ or YPK1 suppressed the resistant phenotype exhibited by the isc1 $\Delta$ mutant, though to a different extent. Deletion of YPK1 in the isc1 $\triangle$ mutant decreased cell survival, but the double mutant strain was still more resistant than wild-type cells. This suggests that the resistance of isc1 $\Delta$ cells to acetic acid partially depends on Ypk1p. However, as far as we know, no association had been described between Ypk1p and the yeast neutral sphingomyelinase Isclp. We therefore sought to determine whether the two proteins physically interact. For this purpose, extracts of BY4741 cells expressing Isc1p-FLAG or vector control were immunoprecipitated with an anti-FLAG antibody, and the presence of Ypklp in extracts and immunoprecipitated fractions was assessed by Western blot (Figure 5).

We found that Ypklp can interact with Isclp, though only a minor amount of total Ypklp was immunoprecipitated by Isc1p-FLAG, suggesting a weak or transient interaction between the two proteins. This indicates that Ypk1p and Isclp may function on the same pathway mediating acetic acid-induced cell death.

On the other hand, double deletion of ISC1 and PKH1 had a surprising drastic effect on cell survival, i.e., the isc1 $\Delta$ $p k h 1 \Delta$ double mutant was much more sensitive to acetic acid than the wild-type strain, even though the single mutants were resistant. We therefore characterized the apoptotic phenotype of isc1 1 pkh1 $1 \Delta$ cells (Figures 6(a) and 6(b)).

We observed that the high sensitivity phenotype of the double mutant was associated with increased superoxide anion accumulation and release of cytochrome $c$ that was not observed in the single-mutant strains. Therefore, simultaneous absence of $\mathrm{PKH} 1$ and ISC1 seemingly overrides the protective effect as a result of individual deletion of $\mathrm{PKH} 1$ or ISC1. As we previously showed [11], we observed that the absence of Isclp led to impaired cytochrome $c$ release, and we further show here that the absence of Pkh1p results in the same phenotype. This suggests that Isclp and Pkh1p are required for cytochrome $c$ release. However, since $p k h 1 \Delta$ isc $1 \Delta$ cells can release cytochrome $c$, it is apparent that there is not an absolute requirement of Isclp or Pkhlp for cytochrome $c$ release, and therefore, the lack of cytochrome $c$ release in individual mutants may be a consequence and not a cause of the increased resistance of those cells. The increased sensitivity of iscl $1 \Delta \mathrm{pkh} 1 \Delta$ cells was nonetheless unexpected, and we therefore investigated the upstream events leading to this phenotype.

\subsection{CWI Signaling Pathway Activation Is Not Involved in the} Sensitivity of isc1 $\triangle$ pkh1 $\triangle$ Cells to Acetic Acid-Induced RCD. During growth and in response to environmental stresses, the cell wall is remodeled in a highly regulated process under the control of the CWI signaling pathway, which facilitates the maintenance of the cell wall by mediating cell wall biosynthesis, actin organization, and other cellular processes [39]. Both the Pkhlp-Ypklp pathway and Isclp were previously implicated in CWI signaling. It was shown that Pkh1p phosphorylates $\mathrm{Pkclp}$, a protein kinase C-like enzyme member of CWI pathway [14], and Ypk1p may contribute to Slt2p activation, leading to activation of the pathway [40]. Additionally, it was found that Isc1p-deficient cells displayed Slt2p hyperphosphorylation and that Slt2p is essential for viability of $i s c 1 \Delta$ cells, since deletion of SLT2 in this mutant results in 


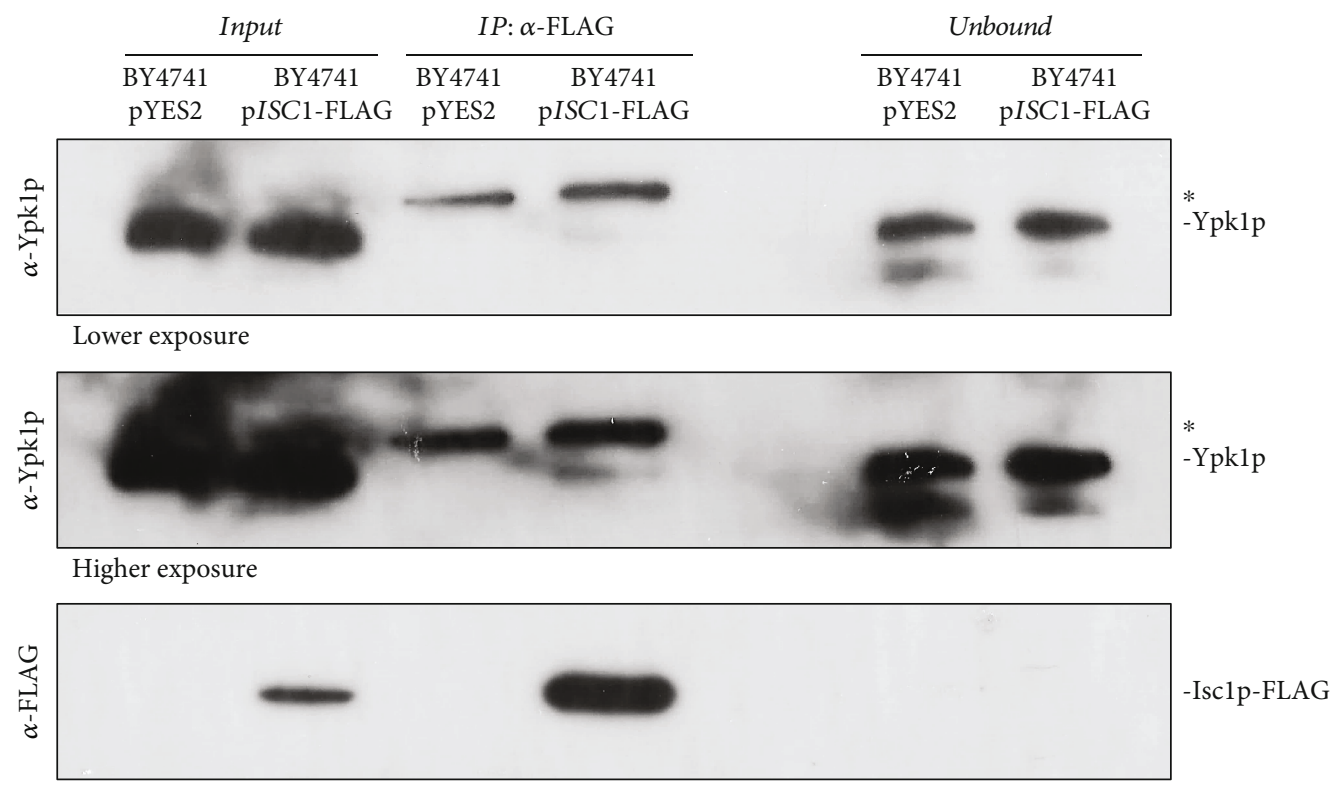

FIGURE 5: Ypk1p interacts with Isc1p. Isc1p-FLAG was immunoprecipitated from protein extracts of BY4741 cells expressing Isc1p-FLAG or vector control grown in SC Gal medium without uracil to exponential phase, and analyzed by Western blot using anti-Ypk1p and antiFLAG antibodies. The band marked with an asterisk $\left({ }^{*}\right)$ represents a non-Ypk1p protein that cross-reacts with the anti-Ypk1p antibody. A lower and a higher exposure of the membranes incubated with anti-Ypklp antibody were displayed to help the visualization of the weak Ypklp band.

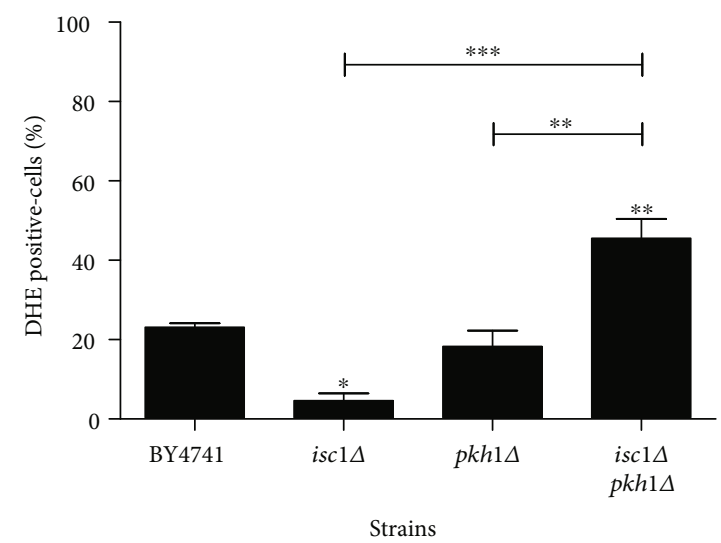

(a)
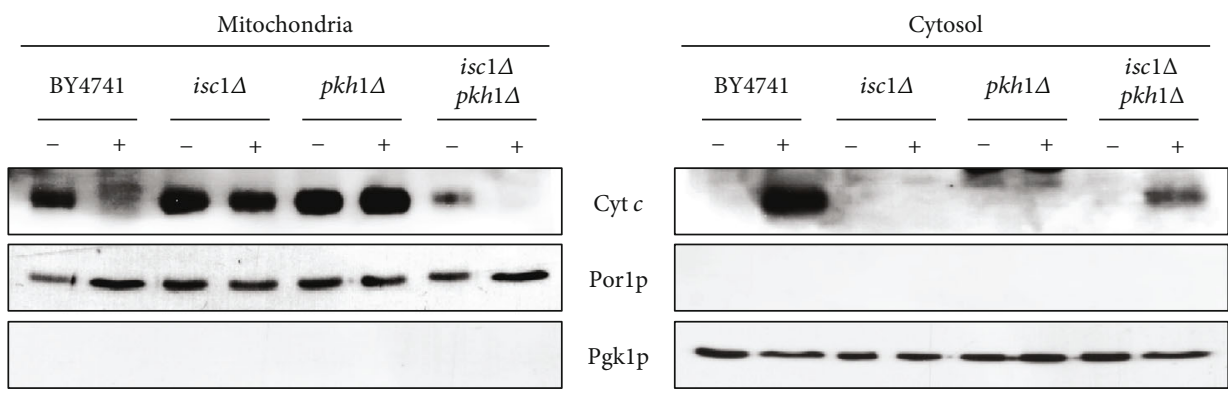

(b)

Figure 6: Sensitivity phenotype of the isc1 $1 \Delta$ ph $1 \Delta$ mutant is associated with increased superoxide anion accumulation and release of cytochrome $c$. (a) Levels of superoxide anion in the indicated strains exposed to acetic acid using DHE. (b) Western blot analysis of cytochrome $c$ before (-) and after (+) exposure to $140 \mathrm{mM}$ acetic acid, $\mathrm{pH}$ 3.0, for $180 \mathrm{~min}$, in both mitochondrial and cytosolic fractions. Cytosolic phosphoglycerate kinase (Pgk1p) and mitochondrial porin (Porlp) levels were used as loading control of cytosolic and mitochondrial fractions, respectively. In (a), values represent mean \pm SD of at least three independent experiments. Values significantly different from BY4741 or between the indicated strains: ${ }^{*} P<0.05,{ }^{* *} P<0.01,{ }^{* * *} P<0.001$, one-way ANOVA, and Tukey's Test. 


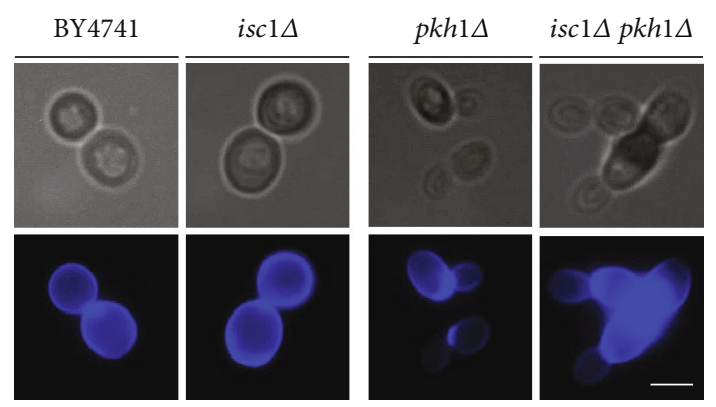

(a)

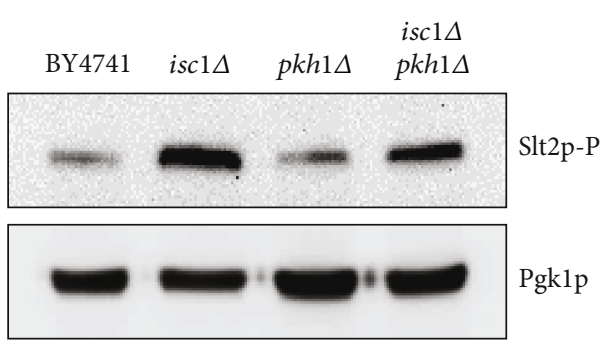

(b)

FIGURE 7: PKH1 deletion increases cell wall defects in the isc1 $\Delta$ mutant strain but does not increase its levels of Slt2p phosphorylation. (a) Cell wall morphology of the indicated strains stained with CFW and observed by fluorescence microscopy. Representative images of two independent experiments are shown. Bar, $5 \mu \mathrm{m}$. (b) Western blot analysis of the basal levels of phosphorylated Slt2p in the indicated strains.

synthetic lethality [33]. The relation of both Pkh1p and Isclp with the CWI pathway led us to investigate whether the sensitivity phenotype of the isc1 $\Delta p k h 1 \Delta$ double mutant could be due to deregulation in the CWI pathway leading to cell wall defects and consequently cell death.

To test this hypothesis, the mutant strains were grown to exponential phase in SC Gal medium and stained with Calcofluor White (CFW) (Figure 7(a)). CFW is a fluorescent stain that binds to structures containing chitin like the yeast cell wall and has been widely used to identify strains with cell wall defects [41]. The CFW stain revealed the presence of chitin in cell walls and its normal accumulation at the bud sites. The morphology of the isc $1 \Delta$ and $p k h 1 \Delta$ strain was quite similar to that of the wild-type strain; however, deletion of both ISC1 and PKH1 resulted in the formation of aberrant cells with elongated buds, wide necks, and large accumulation of chitin in the cells.

The Slt2p MAP kinase is activated by phosphorylation in response to cell wall perturbations. Consistently, our previous results indicated that the CWI pathway is involved in signaling acetic acid-induced cell death, as blocking signal transduction through this pathway renders cells more resistant to acetic acid-induced cell death, and overactivation of the CWI renders cells more sensitive to acetic acid [42]. We therefore investigated whether the high sensitivity of the isc $1 \Delta$ pkh1 $1 \Delta$ double mutant to acetic acid resulted from overactivation of the CWI pathway. As seen in Figure 7(b), Isclp-deficient cells displayed Slt2p hyperphosphorylation as described before [33]. However, despite the basal cell wall defects observed in the isc1 $1 \Delta$ pkh1 $\Delta$ double mutant, we did not observe an increased level of Slt2p phosphorylation in comparison with the isc1 $1 \Delta$ mutant. Thus, both the high resistance and high sensitivity to acetic acid of the isc $1 \Delta$ and isc1 $\Delta$ $p k h 1 \Delta$ double mutants, respectively, are not associated with CWI signaling.

3.5. The Sensitivity of isc $1 \Delta$ pkh1 $1 \Delta$ to Acetic Acid-Induced RCD Depends on $c A M P$ Signaling. The maintenance of the intracellular $\mathrm{pH}$ and the development of a stress response are crucial for yeast cells to survive in an acidic environment. At low $\mathrm{pH}$, acetic acid (undissociated form) enters cells by simple diffusion when grown under glucose-repressed or non-acetic acid permease induction conditions [43]. Another study proposes that the aquaglyceroporin channel Fps1p mediates the transport through a facilitated diffusion process [44]. In any case, once in the cytoplasm and due to a more neutral $\mathrm{pH}$, acetic acid dissociates into acetate and protons, leading to cytoplasmic acidification and inhibition of important metabolic processes which include redox homeostasis, enzymatic activities, or nutrient transport [45-48]. Intracellular acidification has also been described to stimulate the Ras/cAMP/PKA pathway [49, 50]. The levels of cAMP depend on both its synthesis (Ras/adenylate cyclase module) and degradation (cAMP phosphodiesterases) [51]. The yeast cAMP phosphodiesterases Pde1p and Pde2p have low and a high affinity for cAMP, respectively, inhibit PKA by hydrolyzing cAMP and have been shown to be involved in the ability of yeast cells to survive stress conditions [52-54]. Consistently, both cytoplasmic acidification and Ras/cAMP/PKA pathway activation have been described to occur during acetic acid-induced cell death in yeast $[7,55]$. For this reason, we questioned whether the sensitivity phenotype of the isc1 $\Delta$ pkh1 $\Delta$ double mutant could be due to a hyperactivation of the cAMP/PKA pathway in this strain. To test this hypothesis, the wild-type and mutant strains were transformed with a plasmid driving overexpression of PDE2 (pRS426-PDE2) or the empty plasmid (pRS426), grown to early exponential phase in SC Gal medium without uracil, exposed to $140 \mathrm{mM}$ acetic acid, $\mathrm{pH} 3.0$, for $180 \mathrm{~min}$, and characterized regarding acetic acid-induced cell death (Figure 8).

As seen in Figure 8(a), wild-type cells overexpressing $P D E 2$ showed enhanced resistance to acetic acid in comparison with the control strain. In contrast, $P D E 2$ overexpression had no effect on the survival of the isc1 $1 \Delta$ mutant. As observed for the wild-type strain, overexpression of PDE2 increased the survival of $p k h 1 \Delta$ and isc1 $1 p k h 1 \Delta$ mutant cells to acetic acid to levels observed in isc1 $1 \Delta$ mutants. The observation that Pde2p overexpression completely suppresses the sensitivity phenotype of the isc $1 \Delta$ pkh1 $1 \Delta$ double mutant suggests that a hyperactivation of the cAMP/PKA pathway underlies the sensitivity of this strain to acetic acid. Since inappropriate hyperactivation of the CAMP/PKA pathway was also shown to lead to mitochondrial dysfunction, superoxide anion accumulation, and cell death [56], we characterized the phenotype of these cells in more detail (Figures $8(\mathrm{~b})-8(\mathrm{~d})$ ). We found that expression of PDE2 in the isc1 $\triangle$ pkh1 $1 \Delta$ double 


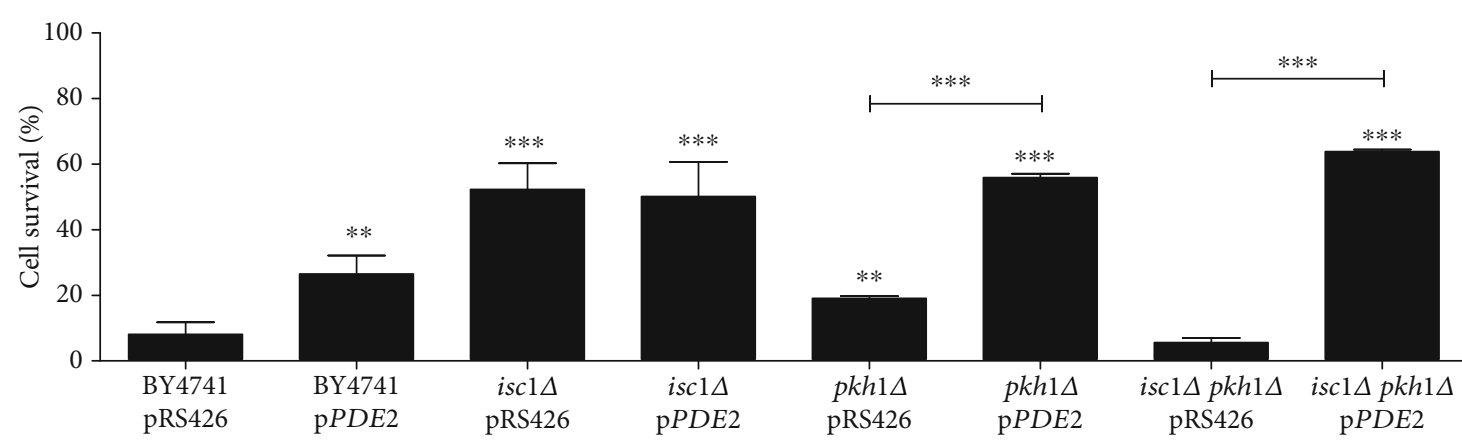

(a)
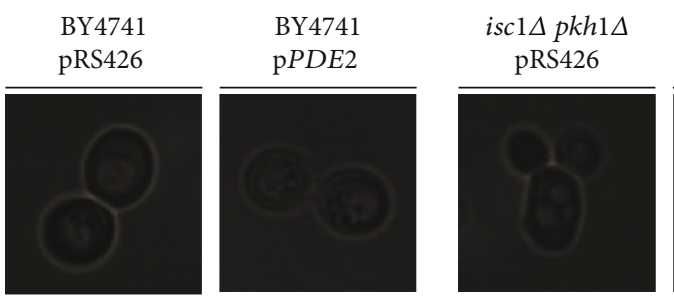

isc $1 \Delta p k h 1 \Delta$

pPDE2
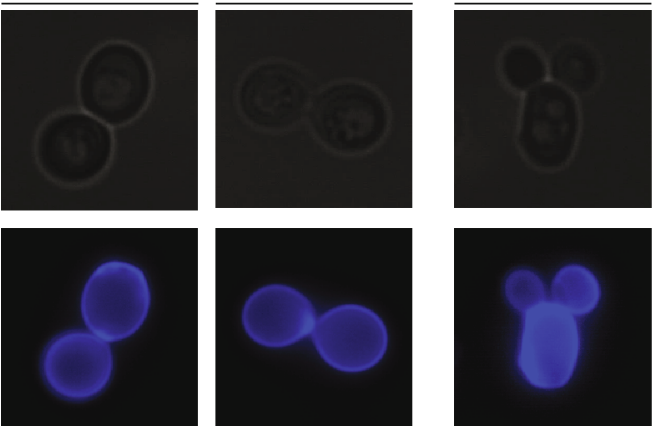

$\mathrm{p} P D E 2$
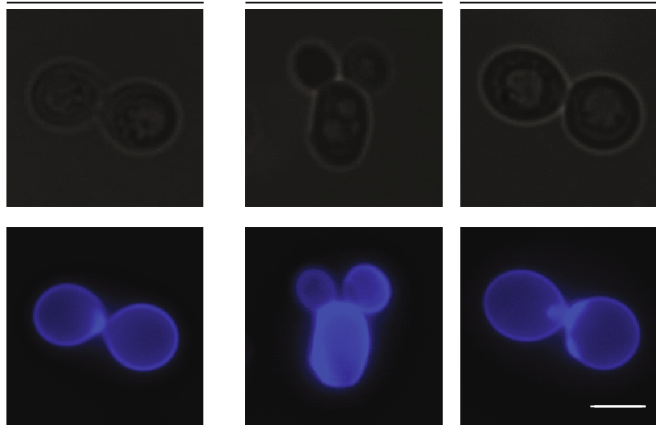

(b)

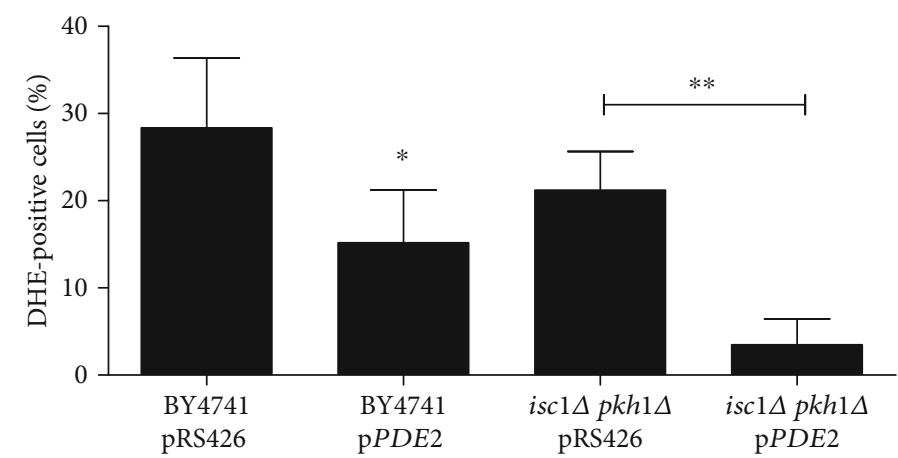

(c)

\begin{tabular}{ccccc}
\multicolumn{4}{c}{ Mitochondria } \\
\hline BY4741 & BY4741 & pkh1 $1 \Delta$ & isc1 \\
pRS426 & $\frac{\mathrm{p} P D E 2}{-+}$ & $\frac{\mathrm{pRS} 426}{-}+$ & $\frac{\mathrm{p} P D E 2}{-}+$ & $\frac{-}{-}+$
\end{tabular}
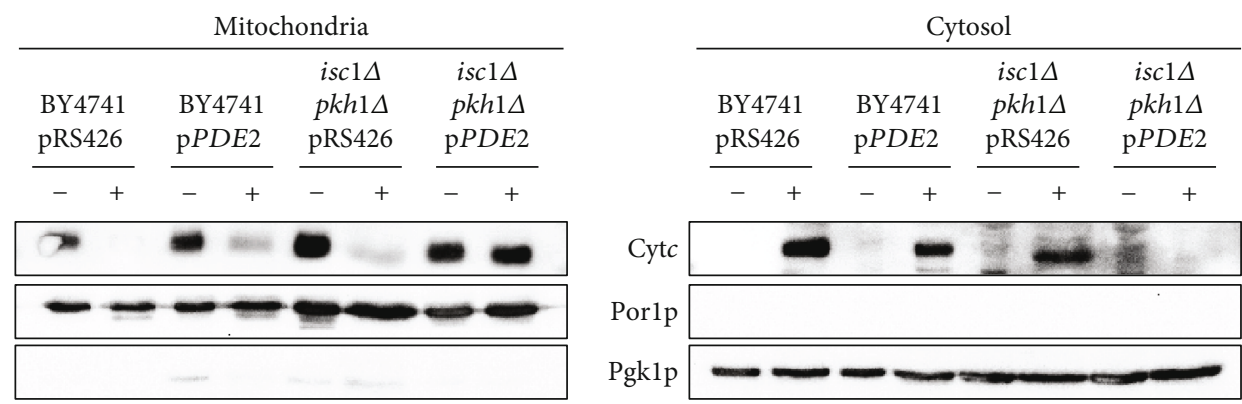

(d)

FIgURE 8: cAMP/PKA pathway is involved in the sensitivity of the isc1 $\Delta$ ph $1 \Delta$ mutant to acetic acid-induced apoptosis. (a) Cell survival of the indicated strains to $140 \mathrm{mM}$ of acetic acid, $\mathrm{pH} 3.0$, for $180 \mathrm{~min}$. (b) Cell wall morphology of the indicated strains stained with CFW and observed by fluorescence microscopy. Representative images of two independent experiments are shown. Bar, $5 \mu \mathrm{m}$. (c) Levels of superoxide anion in the indicated strains exposed to acetic acid using DHE. (c) Western blot analysis of cytochrome $c$ before (-) and after (+) exposure to $140 \mathrm{mM}$ acetic acid, pH3.0, for $180 \mathrm{~min}$, in both mitochondrial and cytosolic fractions. Cytosolic phosphoglycerate kinase (Pgk1p) and mitochondrial porin (Porlp) levels were used as loading control of cytosolic and mitochondrial fractions, respectively. In (a) and (c), values represent mean \pm SD of at least three independent experiments. Values significantly different from BY4741 pRS426 or between the indicated strains: ${ }^{*} P<0.05,{ }^{* *} P<0.01,{ }^{* * *} P<0.001$, one-way ANOVA, and Tukey's Test. 
mutant abolished the formation of aberrant cells and large accumulation of chitin observed in isc1 $\Delta$ pkh1 $\Delta$ cells, and decreased the superoxide anion accumulation and cytochrome $c$ release in response to acetic acid. These results indicate that Pkhpl and Isclp regulate cAMP levels through a yet uncharacterized mechanism and that the increased sensitivity of $i s c 1 \Delta p k h 1 \Delta$ cells to acetic acid is due to abnormally increased cAMP levels.

\section{Conclusions}

In this study, the relative contribution of several signaling pathways and how sphingolipid signaling regulates acetic acid-induced cell death, possibly through Isclp as a downstream target, was determined. We first showed that Pkh1p and Ypklp have a pro-death role, similarly to Isclp and Sch9p [28]. We also found that acetic acid exposure leads to Pkh1/2p-dependent phosphorylation of both Sch9p and Ypk1p, indicating that Pkh1p-Ypk1p and Pkh1p-Sch9p pathways are activated during acetic acid-induced cell death. Since we had previously described that Sch9p is involved in Isclp translocation to mitochondria, the role of the PkhlpYpk1p pathway in the resistance of isc1 $\Delta$ was addressed in more detail. We show that deletion of either YPK1 or PKH1 leads to a decrease in the survival of isc1 $\Delta$ mutants exposed to acetic acid. However, while isc1 $\Delta$ pk $1 \Delta$ cells were still more resistant than wild-type, double deletion of $P K H 1$ and ISC1 resulted in hypersensitivity, leading to the emergence of apoptotic markers such as superoxide anion accumulation and cytochrome $c$ release. This was correlated with presumed increased cAMP levels, as it was reverted by overexpression of Pde2p. Indeed, the resistant phenotypes of single (isc1 $\Delta$ or $p k h 1 \Delta$ ) and the double (isc1 $1 \Delta$ ph1 $1 \Delta$ ) mutants overexpressing Pde 2 p were very similar, which indicates that Pkh1p and Isclp play a role in the mediation of acetic acid-induced cell death under those conditions. Therefore, the sensitive phenotype of isc $1 \Delta p k h 1 \Delta$ cells is likely an indirect consequence of increased cAMP levels, which leads to acetic acid sensitivity, and not a result of a direct protective role of either Pkh1p or Isc1p in acetic acid-induced cell death.

Taken together, our results suggest that both PkhlpYpk1p and Pkh1p-Sch9p pathways play a role in Isc1pmediated acetic acid-induced cell death. Sch9p seems to be important for Isclp activation and translocation into mitochondria [28]. As we observed that Ypk1p may interact with Isc1p, it will be interesting in the future to determine whether Ypk1p and/or Pkh1p are involved in translocation of Isc1p, as observed for Sch9p [28]. On the other hand, it has been reported that Ypk1p is responsible for the phosphorylation of Laclp and Laglp ceramide synthases [26]. Therefore, the Pkh1p-Ypklp pathway might also be involved in the activation of Laglp in acetic acid-induced cell death in wild-type cells. In accordance with the hypothesis that Ypklp activates Lag1p, we have previously shown that the deletion of LAG1 increases the cellular resistance of yeasts to acetic acidinduced cell death [11]. Another study on acetic acid tolerance already showed that Ypk1p, being activated by Torc2p, phosphorylates Laglp and Laclp [57]. Further studies to decipher the link between the Pkh1p-Ypk1p pathway and ceramide de novo biosynthesis should be performed.

Overall, our results suggest that Pkh1p-Ypk1p and Pkh1p-Sch9p pathways are activated during acetic acidinduced cell death. Since the importance of ceramide in mammalian cell death is increasingly apparent, our study further supports the use of yeast as a valuable model to investigate the modulation of regulated cell death by ceramide.

\section{Data Availability}

The data used to support the findings of this study are included within the article and the supplementary information file.

\section{Conflicts of Interest}

The authors declare no conflict of interests.

\section{Authors' Contributions}

AR, FM, SRC, VC, and MCR conceived and designed the experiments. AR and FM performed the experiments. AR, FM, SRC, VC, and MCR analyzed the data. VC and MCR contributed with reagents/materials/analysis tools. AR, SRC, VC, and MCR wrote the paper. Susana Chaves and Manuela Côrte-Real contributed equally to this work.

\section{Acknowledgments}

We thank Dr. Maria João Sousa for helpful discussions, Dr. Cory Dunn for pRS426 and pRS426-PDE2 plasmids [58], Dr. Jeremy Thorner for pRS316-YPK1, pRS316-YPK1 (T504D), pRS315-SCH9, and pRS315-SCH9 (T570A) plasmids [18], Dr. Robert Dickson for the anti-Sch9p antibody, Dr. Robbie Loewith for the anti-Phospho-T570-Sch9p, and Dr. Isabel Alonso for the mouse IgG-peroxidase TrueBlot $^{\circledR}$ ULTRA antibody. This project was financially supported by Fundação para a Ciência e Tecnologia (FCT) by the projects: PestC/BIA/UI4050/2013 (POCI-01-0145-FEDER-007569), PEstOE/BIA/UI4050/2014, FCOMP-01-0124-FEDER-028210 (PTDC/BBB-BQB/1850/2012), FCOMP-01-0124-FEDER022718 (PEst-C/SAU/LA0002/2011), FCT-ANR/BEXBCM/ 0175/2012, and UID/BIM/04293/2013, as well as a post doc fellowship to S. Chaves (SFRH/BPD/89980/2012), a PhD fellowship to A. Rego (SFRH/BD/79523/2011), and a PhD fellowship to F. Mendes (SFRD/BD/147574/2019).

\section{Supplementary Materials}

Figure S1: percentage of PI-positive wild-type cells after $180 \mathrm{~min}$ of acetic acid exposure (treatment) and $120 \mathrm{~min}$ and $240 \mathrm{~min}$ after transfer to fresh medium. BY4741 cells were grown in Synthetic Complete Galactose (SC Gal) medium to early exponential phase at $26^{\circ} \mathrm{C}$ and $200 \mathrm{rpm}$, harvested, and suspended in SC Gal at pH3.0 without or with $140 \mathrm{mM}$ acetic acid (AA). After $180 \mathrm{~min}$ of treatment, plasma membrane integrity was assessed by flow cytometry using propidium iodide (PI) staining. PI was added to yeast cell suspensions to a final concentration of $5 \mu \mathrm{g} / \mathrm{mL}$ and incubated 
for $10 \mathrm{~min}$ at room temperature. Next, the remaining cells were harvested, washed, suspended in fresh SC Gal medium, and incubated at $26^{\circ} \mathrm{C}$ and $200 \mathrm{rpm}$. After $120 \mathrm{~min}$ and $240 \mathrm{~min}$, plasma membrane integrity was assessed as described before. Values represent mean $\pm \mathrm{SD}$ of at least three independent experiments. Values significantly different from the BY4741 control strain: ${ }^{* * *} P<0.001$, Oneway ANOVA, and Tukey Test. Figure S2: basal O2 consumption rates of the of wild-type strain in the absence and presence of the uncoupler Carbonyl Cyanide-ptrifluoromethoxyphenylhydrazone (FCCP) and the respiratory chain inhibitors sodium azide and Antimycin A. BY4741 cells were grown in Synthetic Complete Galactose (SC Gal) medium to early exponential phase at $26^{\circ} \mathrm{C}$ and $200 \mathrm{rpm}$, then harvested, and suspended in water $\left(\mathrm{OD}_{600 \mathrm{~nm}}=20\right)$. A volume of $0.4 \mathrm{~mL}$ of yeast suspension was added to the chamber with $4.6 \mathrm{~mL}$ of deionized water and stirred with a magnetic bar, and the oxygen consumption was recorded with a Clark electrode. FCCP and sodium azide were added to yeast cell suspensions in the chamber to a final concentration of 1 and $5 \mu \mathrm{M}$, and Antimiycin A to 0.5 and $1 \mathrm{nM}$. The oxygen consumption rate values were calculated from the slopes of the initial part of the oxygen consumption trace and normalized to the dry weight of the cell culture. Values represent mean \pm SD of at least three independent experiments. Values significantly different from the BY4741 control strain: ${ }^{* * *} P<0.001$, one-way ANOVA, and Tukey's Test. (Supplementary Materials)

\section{References}

[1] R. C. Dickson, "Roles for sphingolipids in Saccharomyces cerevisiae," Advances in Experimental Medicine and Biology, vol. 688, pp. 217-231, 2010.

[2] Y. A. Hannun and L. M. Obeid, "Principles of bioactive lipid signalling: lessons from sphingolipids," Nature Reviews Molecular Cell Biology, vol. 9, no. 2, pp. 139-150, 2008.

[3] F. B. Ozbayraktar and K. O. Ulgen, "Molecular facets of sphingolipids: mediators of diseases," Biotechnology Journal, vol. 4, no. 7, pp. 1028-1041, 2009.

[4] A. Rego, D. Trindade, S. R. Chaves et al., "The yeast model system as a tool towards the understanding of apoptosis regulation by sphingolipids," FEMS Yeast Research, vol. 14, no. 1, pp. 160-178, 2014.

[5] B. J. Pettus, C. E. Chalfant, and Y. A. Hannun, "Ceramide in apoptosis: an overview and current perspectives," Biochimica et Biophysica Acta, vol. 1585, no. 2-3, pp. 114-125, 2002.

[6] P. Ludovico, F. Rodrigues, A. Almeida, M. T. Silva, A. Barrientos, and M. Corte-Real, "Cytochrome $c$ release and mitochondria involvement in programmed cell death induced by acetic acid in Saccharomyces cerevisiae," Molecular Biology of the Cell, vol. 13, no. 8, pp. 2598-2606, 2002.

[7] P. Ludovico, M. J. Sousa, M. T. Silva, C. Leao, and M. CorteReal, "Saccharomyces cerevisiae commits to a programmed cell death process in response to acetic acid," Microbiology, vol. 147, Part 9, pp. 2409-2415, 2001.

[8] N. Guaragnella, S. Passarella, E. Marra, and S. Giannattasio, "Knock-out of metacaspase and/or cytochrome c results in the activation of a ROS-independent acetic acid-induced pro- grammed cell death pathway in yeast," FEBS Letters, vol. 584, no. 16, pp. 3655-3660, 2010.

[9] N. Guaragnella, C. Pereira, M. J. Sousa et al., "YCA1 participates in the acetic acid induced yeast programmed cell death also in a manner unrelated to its caspase-like activity," FEBS Letters, vol. 580, no. 30, pp. 6880-6884, 2006.

[10] N. Guaragnella, M. Zdralevic, L. Antonacci, S. Passarella, E. Marra, and S. Giannattasio, "The role of mitochondria in yeast programmed cell death," Frontiers in Oncology, vol. 2, p. 70, 2012.

[11] A. Rego, M. Costa, S. R. Chaves et al., "Modulation of mitochondrial outer membrane permeabilization and apoptosis by ceramide metabolism," PLoS One, vol. 7, no. 11, article e48571, 2012.

[12] A. Casamayor, P. D. Torrance, T. Kobayashi, J. Thorner, and D. R. Alessi, "Functional counterparts of mammalian protein kinases PDK1 and SGK in budding yeast," Current Biology, vol. 9, no. 4, pp. 186-197, 1999.

[13] S. Friant, R. Lombardi, T. Schmelzle, M. N. Hall, and H. Riezman, "Sphingoid base signaling via Pkh kinases is required for endocytosis in yeast," The EMBO Journal, vol. 20, no. 23, pp. 6783-6792, 2001.

[14] M. Inagaki, T. Schmelzle, K. Yamaguchi, K. Irie, M. N. Hall, and K. Matsumoto, "PDK1 homologs activate the Pkc1mitogen-activated protein kinase pathway in yeast," Molecular and Cellular Biology, vol. 19, no. 12, pp. 8344-8352, 1999.

[15] K. Liu, X. Zhang, R. L. Lester, and R. C. Dickson, "The sphingoid long chain base phytosphingosine activates AGC-type protein kinases in Saccharomyces cerevisiae including Ypk 1, Ypk 2, and Sch 9," The Journal of Biological Chemistry, vol. 280, no. 24, pp. 22679-22687, 2005.

[16] F. M. Roelants, P. D. Torrance, N. Bezman, and J. Thorner, "Pkh 1 and Pkh 2 differentially phosphorylate and activate Ypk 1 and Ykr 2 and define protein kinase modules required for maintenance of cell wall integrity," Molecular Biology of the Cell, vol. 13, no. 9, pp. 3005-3028, 2002.

[17] B. J. Niles, H. Mogri, A. Hill, A. Vlahakis, and T. Powers, "Plasma membrane recruitment and activation of the AGC kinase Ypk 1 is mediated by target of rapamycin complex 2 (TORC2) and its effector proteins Slm 1 and SIm 2," Proceedings of the National Academy of Sciences, vol. 109, no. 5, pp. 1536-1541, 2012.

[18] F. M. Roelants, P. D. Torrance, and J. Thorner, "Differential roles of PDK1- and PDK2-phosphorylation sites in the yeast AGC kinases Ypk 1, Pkc 1 and Sch 9," Microbiology, vol. 150, Part 10, pp. 3289-3304, 2004.

[19] K. Voordeckers, M. Kimpe, S. Haesendonckx, W. Louwet, M. Versele, and J. M. Thevelein, "Yeast 3-phosphoinositidedependent protein kinase-1 (PDK1) orthologs Pkh1-3 differentially regulate phosphorylation of protein kinase a (PKA) and the protein kinase B (PKB)/S6K ortholog Sch9," The Journal of Biological Chemistry, vol. 286, no. 25, pp. 22017-22027, 2011.

[20] J. Urban, A. Soulard, A. Huber et al., "Sch 9 is a major target of TORC1 in Saccharomyces cerevisiae," Molecular Cell, vol. 26, no. 5, pp. 663-674, 2007.

[21] A. Pascual-Ahuir and M. Proft, "Control of stress-regulated gene expression and longevity by the Sch 9 protein kinase," Cell Cycle, vol. 6, no. 20, pp. 2445-2447, 2007.

[22] J. T. Nickels and J. R. Broach, "A ceramide-activated protein phosphatase mediates ceramide-induced G1 arrest of 
Saccharomyces cerevisiae," Genes \& Development, vol. 10, no. 4, pp. 382-394, 1996.

[23] C. J. Di Como and K. T. Arndt, "Nutrients, via the Tor proteins, stimulate the association of tap 42 with type $2 \mathrm{~A}$ phosphatases," Genes \& Development, vol. 10, no. 15, pp. 1904-1916, 1996.

[24] M. Liu, C. Huang, S. R. Polu, R. Schneiter, and A. Chang, "Regulation of sphingolipid synthesis through Orm 1 and Orm 2 in yeast," Journal of Cell Science, vol. 125, Part 10, pp. 2428-2435, 2012.

[25] M. Shimobayashi, W. Oppliger, S. Moes, P. Jeno, and M. N. Hall, “TORC1-regulated protein kinase Npr 1 phosphorylates Orm to stimulate complex sphingolipid synthesis," Molecular Biology of the Cell, vol. 24, no. 6, pp. 870-881, 2013.

[26] A. Muir, S. Ramachandran, F. M. Roelants, G. Timmons, and J. Thorner, "TORC2-dependent protein kinase Ypk 1 phosphorylates ceramide synthase to stimulate synthesis of complex sphingolipids," Elife, vol. 3, 2014.

[27] E. Swinnen, T. Wilms, J. Idkowiak-Baldys et al., “The protein kinase Sch 9 is a key regulator of sphingolipid metabolism in Saccharomyces cerevisiae," Molecular Biology of the Cell, vol. 25, no. 1, pp. 196-211, 2014.

[28] A. Rego, K. F. Cooper, J. Snider et al., "Acetic acid induces Sch9p-dependent translocation of Isclp from the endoplasmic reticulum into mitochondria," Biochimica et Biophysica Acta Molecular and Cell Biology of Lipids, vol. 1863, no. 6, pp. 576$583,2018$.

[29] R. D. Gietz and R. A. Woods, "Transformation of yeast by lithium acetate/single-stranded carrier DNA/polyethylene glycol method," Methods in Enzymology, vol. 350, pp. 87-96, 2002.

[30] P. Herrero, R. Fernandez, and F. Moreno, "Differential sensitivities to glucose and galactose repression of gluconeogenic and respiratory enzymes from Saccharomyces cerevisiae," Archives of Microbiology, vol. 143, no. 3, pp. 216-219, 1985.

[31] A. D. Barbosa, H. Osorio, K. J. Sims et al., "Role for Sit4pdependent mitochondrial dysfunction in mediating the shortened chronological lifespan and oxidative stress sensitivity of Isclp-deficient cells," Molecular Microbiology, vol. 81, no. 2, pp. 515-527, 2011.

[32] T. Almeida, M. Marques, D. Mojzita et al., "Isc1p plays a key role in hydrogen peroxide resistance and chronological lifespan through modulation of iron levels and apoptosis," Molecular Biology of the Cell, vol. 19, no. 3, pp. 865-876, 2008.

[33] A. D. Barbosa, J. Graca, V. Mendes et al., "Activation of the Hoglp kinase in Isc1p-deficient yeast cells is associated with mitochondrial dysfunction, oxidative stress sensitivity and premature aging," Mechanisms of Ageing and Development, vol. 133, no. 5, pp. 317-330, 2012.

[34] V. Teixeira, T. C. Medeiros, R. Vilaca, J. Ferreira, P. MoradasFerreira, and V. Costa, "Ceramide signaling targets the PP2Alike protein phosphatase Sit4p to impair vacuolar function, vesicular trafficking and autophagy in Isc1p deficient cells," Biochimica et Biophysica Acta, vol. 1861, no. 1, pp. 21-33, 2016.

[35] V. Teixeira, T. C. Medeiros, R. Vilaca, P. Moradas-Ferreira, and V. Costa, "Reduced TORC1 signaling abolishes mitochondrial dysfunctions and shortened chronological lifespan of Isc1p-deficient cells," Microbial Cell, vol. 1, no. 1, pp. 21-36, 2014.

[36] N. D. Bonawitz, M. Chatenay-Lapointe, Y. Pan, and G. S. Shadel, "Reduced TOR signaling extends chronological life span via increased respiration and upregulation of mitochondrial gene expression," Cell Metabolism, vol. 5, no. 4, pp. 265-277, 2007.

[37] P. Zabrocki, C. Van Hoof, J. Goris, J. M. Thevelein, J. Winderickx, and S. Wera, "Protein phosphatase 2A on track for nutrient-induced signalling in yeast," Molecular Microbiology, vol. 43, no. 4, pp. 835-842, 2002.

[38] F. M. Roelants, A. G. Baltz, A. E. Trott, S. Fereres, and J. Thorner, "A protein kinase network regulates the function of aminophospholipid flippases," Proceedings of the National Academy of Sciences, vol. 107, no. 1, pp. 34-39, 2010.

[39] D. E. Levin, "Cell wall integrity signaling in Saccharomyces cerevisiae," Microbiology and Molecular Biology Reviews, vol. 69, no. 2, pp. 262-291, 2005.

[40] T. Schmelzle, S. B. Helliwell, and M. N. Hall, "Yeast protein kinases and the RHO1 exchange factor TUS1 are novel components of the cell integrity pathway in yeast," Molecular and Cellular Biology, vol. 22, no. 5, pp. 1329-1339, 2002.

[41] A. F. Ram and F. M. Klis, "Identification of fungal cell wall mutants using susceptibility assays based on Calcofluor white and Congo red," Nature Protocols, vol. 1, no. 5, pp. 22532256, 2006.

[42] A. Rego, A. Duarte, F. Azevedo, M. Sousa, M. Côrte-Real, and S. Chaves, "Cell wall dynamics modulate acetic acid-induced apoptotic cell death of Saccharomyces cerevisiae," Microbial Cell, vol. 1, no. 9, pp. 303-314, 2014.

[43] M. Casal, H. Cardoso, and C. Leao, "Mechanisms regulating the transport of acetic acid in Saccharomyces cerevisiae," Microbiology, vol. 142, no. 6, pp. 1385-1390, 1996.

[44] M. Mollapour and P. W. Piper, "Hog 1 mitogen-activated protein kinase phosphorylation targets the yeast Fps 1 aquaglyceroporin for endocytosis, thereby rendering cells resistant to acetic acid," Molecular and Cellular Biology, vol. 27, no. 18, pp. 6446-6456, 2007.

[45] L. U. Guldfeldt and N. Arneborg, "Measurement of the effects of acetic acid and extracellular $\mathrm{pH}$ on intracellular $\mathrm{pH}$ of nonfermenting, individual Saccharomyces cerevisiae cells by fluorescence microscopy," Applied and Environmental Microbiology, vol. 64, no. 2, pp. 530-534, 1998.

[46] M. E. Pampulha and M. C. Loureiro-Dias, "Combined effect of acetic acid, $\mathrm{pH}$ and ethanol on intracellular $\mathrm{pH}$ of fermenting yeast," Applied Microbiology and Biotechnology, vol. 31-31, no. 5-6, pp. 547-550, 1989.

[47] M. E. Pampulha and M. C. Loureiro-Dias, "Energetics of the effect of acetic acid on growth of Saccharomyces cerevisiae," FEMS Microbiology Letters, vol. 184, no. 1, pp. 69-72, 2000.

[48] N. Arneborg, L. Jespersen, and M. Jakobsen, "Individual cells of Saccharomyces cerevisiae and Zygosaccharomyces bailii exhibit different short-term intracellular $\mathrm{pH}$ responses to acetic acid," Archives of Microbiology, vol. 174, no. 1-2, pp. $125-128,2000$.

[49] J. M. Thevelein, "Fermentable sugars and intracellular acidification as specific activators of the RAS-adenylate cyclase signalling pathway in yeast: the relationship to nutrient-induced cell cycle control," Molecular Microbiology, vol. 5, no. 6, pp. 1301-1307, 1991.

[50] J. M. Thevelein, M. Beullens, F. Honshoven et al., "Regulation of the cAMP level in the yeast Saccharomyces cerevisiae: the glucose-induced cAMP signal is not mediated by a transient drop in the intracellular pH," Journal of General Microbiology, vol. 133, no. 8, pp. 2197-2205, 1987. 
[51] J. I. Park, C. M. Grant, and I. W. Dawes, "The high-affinity cAMP phosphodiesterase of Saccharomyces cerevisiae is the major determinant of cAMP levels in stationary phase: involvement of different branches of the Ras-cyclic AMP pathway in stress responses," Biochemical and Biophysical Research Communications, vol. 327, no. 1, pp. 311-319, 2005.

[52] P. Ma, S. Wera, P. Van Dijck, and J. M. Thevelein, “The PDE1-encoded low-affinity phosphodiesterase in the yeast Saccharomyces cerevisiae has a specific function in controlling agonist-induced cAMP signaling," Molecular Biology of the Cell, vol. 10, no. 1, pp. 91-104, 1999.

[53] J. Nikawa, P. Sass, and M. Wigler, "Cloning and characterization of the low-affinity cyclic AMP phosphodiesterase gene of Saccharomyces cerevisiae," Molecular and Cellular Biology, vol. 7, no. 10, pp. 3629-3636, 1987.

[54] P. Sass, J. Field, J. Nikawa, T. Toda, and M. Wigler, "Cloning and characterization of the high-affinity cAMP phosphodiesterase of Saccharomyces cerevisiae," Proceedings of the National Academy of Sciences, vol. 83, no. 24, pp. 9303-9307, 1986.

[55] A. J. Phillips, J. D. Crowe, and M. Ramsdale, "Ras pathway signaling accelerates programmed cell death in the pathogenic fungus Candida albicans," Proceedings of the National Academy of Sciences, vol. 103, no. 3, pp. 726-731, 2006.

[56] J. E. Leadsham and C. W. Gourlay, "cAMP/PKA signaling balances respiratory activity with mitochondria dependent apoptosis via transcriptional regulation," BMC Cell Biology, vol. 11 , no. 1 , p. $92,2010$.

[57] J. F. Guerreiro, A. Muir, S. Ramachandran, J. Thorner, and I. Sa-Correia, "Sphingolipid biosynthesis upregulation by TOR complex 2-Ypk1 signaling during yeast adaptive response to acetic acid stress," The Biochemical Journal, vol. 473, no. 23, pp. 4311-4325, 2016.

[58] E. Akdogan, M. Tardu, G. Garipler, G. Baytek, I. H. Kavakli, and C. D. Dunn, "Reduced glucose sensation can increase the fitness of Saccharomyces cerevisiae lacking mitochondrial DNA," PLoS One, vol. 11, no. 1, article e0146511, 2016. 